\title{
Frequent aberrant p53 and Fhit expression in endoscopically resected superficial hypopharyngeal cancer and esophageal cancer
}

\author{
SOHEI YAMAMOTO, KAZUO YASHIMA, SOICHIRO KAWATA, KOHEI HOSODA, \\ AKIHIRO TAMOTO, YUICHIRO IKEBUCHI, KAZUYA MATSUMOTO, KOICHIRO KAWAGUCHI, \\ KENICHI HARADA, YOSHIKAZU MURAWAKI and HAJIME ISOMOTO
}

Division of Medicine and Clinical Science, Faculty of Medicine, University of Tottori, Yonago 683-8504, Japan

Received October 18, 2015; Accepted February 17, 2017

DOI: $10.3892 / \mathrm{ol} .2017 .6271$

\begin{abstract}
In the last decade, the incidence rate of detection rate of superficial head, neck and esophageal squamous cell carcinomas has increased with the development of endoscopic imaging techniques. These cancers are thought to arise independently subsequent to tissue exposure to a common carcinogen e.g. alcohol or tobacco. This phenomenon has been termed field cancerization. To determine the molecular background of the development of hypopharyngeal squamous cell carcinomas (HPSCCs) and double esophageal squamous cell carcinomas (DESCCs), the present study immunohistochemically assessed tumor-related protein expression [p53, Fhit (fragile histidine triad), E-cadherin and activation-induced cytidine deaminase (AID)], and subsequently determined the correlation between protein expression and clinicopathological data. Tumor specimens of 9 HPSCCs and 9 DESCCs were endoscopically obtained from 8 patients with HPSCC. The 9 DESCCs, including 5 synchronous and 4 metachronous lesions, were all obtained from four patients with HPSCC. The overexpression of p53 and loss of Fhit expression was immunohistochemically detected in $8(88.9 \%)$ and $8(88.9 \%)$ of the 9 HPSCCs and in $8(88.9 \%)$ and $8(88.9 \%)$ of the 9 DESCCs, respectively, which demonstrated the high frequency of such expression. Additionally, 7 out of 9 HPSCCs, and 7 out of 9 DESCCs demonstrated aberrant expression of p53 and Fhit. The rate of aberrant AID and E-cadherin expression was 67 and $44 \%$ in HPSCCs and 44 and 44\% in DESCC, respectively. These results suggested that aberrant p53 and Fhit expression was involved in the development of HPSCC and their DESCC, and that their expression may be used for the prediction of DESCC development in patients with HPSCC, thereby acting as a biomarker of field cancerization.
\end{abstract}

Correspondence to: Dr Kazuo Yashima, Division of Medicine and Clinical Science, Faculty of Medicine, University of Tottori, 36-1 Nishicho, Yonago 683-8504, Japan

E-mail: yashima@med.tottori-u.ac.jp

Key words: hypopharyngeal cancer, esophageal cancer, endoscopic resection, p53, fragile histidine triad

\section{Introduction}

Cancers of the head, neck and esophagus are associated with the same carcinogens, in particular tobacco and alcohol $(1,2)$. Smoking and alcohol consumption have been associated with an increase in the rate of incidence of synchronous or metachronous cancers in patients with head and neck cancer (HNC). The independent development of such cancers following exposure to a common carcinogen is known as the field cancerization phenomenon (3). In particular, synchronous or metachronous esophageal cancer has been frequently detected in patients with HNC (4).

The majority of hypopharyngeal squamous cell carcinomas (HPSCCs) have a poor prognosis. One reason for this poor prognosis is that early detection of HPSCC is often difficult since Lugol chromoendoscopy is used in the esophagus but not at the laryngopharyngeal site (5). HPSCCs are therefore often found as advanced disease at the initial diagnosis (6). However, recent studies suggested that superficial squamous neoplasms in the orohypopharynx and esophagus can be diagnosed with a high diagnostic value using narrow-band imaging (NBI) endoscopy with or without magnification (6). In addition, previous Japanese studies suggest that peroral endoscopic resection is useful for treatment of superficial HPSCC, in which tumor depth is confined to the subepithelial layer $(7,8)$.

Understanding the molecular events that underlie the development of HPSCC and its double esophageal squamous cell carcinoma (DESCC) may aid the development of strategies for the prevention and therapy of these cancers $(1,2)$. These findings may also be useful in developing diagnostic tests for patients with second primary malignancies. It has been reported that carcinogenesis and its progression in head, neck and esophageal cancers involves the abnormal expression of several genes including p53, Fragile Histidine Triad (FHIT) and E-cadherin $(1,9,10)$. However, little is known regarding whether these genes are abnormally expressed in endoscopically resected specimens of superficial HPSCCs and their DESCC.

p53 is a tumor-suppressor gene, which can be mutated by tobacco smoke and alcohol consumption (2). Furthermore, Helicobacter pylori-mediated upregulation of the DNA- and RNA-editing enzyme, activation-induced cytidine deaminase (AID) (11), was recently reported to result in the accumulation 
of nucleotide alterations in the p53 tumor suppressor gene in gastric cells in vitro (12). The protein encoded by the p53 gene is essential for growth suppression, apoptosis and DNA repair (13). Modulation of the FHIT gene, which is located in the most active human fragile region, FRA3B (14), and of Fhit protein expression, has also been linked to ESCC and cigarette smoking. Thus, Fhit is inactivated at an early stage in the development of ESCC (15); FHIT gene promoter methylation has been linked to cigarette smoking $(16,17)$, and ESCC may be associated with loss of the Fhit protein expression along with alcohol consumption $(15,18)$. Fhit protein expression has been reported to be associated with growth inhibition and the induction of apoptosis (19). Loss of E-cadherin expression has been reported to correlate with tumor invasiveness, metastasis and prognosis of head, neck and esophageal cancer $(9,20)$. E-cadherin is an important cell-to-cell adhesion molecule that is essential for the development and maintenance of cell polarity and tissue architecture (21).

The purpose of the present study was to test the field cancerization hypothesis at the molecular level by identifying aberrant tumor-related protein expression in superficial HPSCCs and ESCCs arising in the same patient. To assess biological properties, the expression of p53, Fhit, E-cadherin and AID were immunohistochemically examined in endoscopically resected HPSCCs and their synchronous or metachronous secondary ESCCs.

\section{Patients and methods}

Patient and tissue samples. A total of 18 tumor specimens, consisting of 9 HPSCCs and 9 DESCCs, were endoscopically resected from 8 patients with HPSCC at the University of Tottori (Yonago, Japan) between January 2010 and December 2014. The 9 DESCCs, including 5 synchronous and 4 metachronous cancers, were obtained from 4 patients with HPSCC. Synchronous or metachronous neoplasms were defined according to the criteria proposed by Warren and Gates (22). Thus, a synchronous carcinoma was defined as a second neoplasm that was diagnosed concurrently or within 6 months of the primary lesion diagnosis. Prior to or subsequent to this period, neoplasms were considered metachronous. Patient anonymity was maintained by assigning all specimens a unique number and excluding all personal information. The present study was approved by the Institutional Ethics Committee of Tottori University (no. 314 and 1508A024) and complied with the Declaration of Helsinki. Written informed consent was obtained from all 8 patients involved in the present study.

Analysis of tobacco and alcohol involvement. The Brinkman index (BI) was used to calculate the patients' history of smoking and is defined as the number of cigarettes per day $\mathrm{x}$ number of years of smoking. The drinking index (DI) was used to calculate the patients' history of drinking and the accumulated amount of alcohol. DI was defined as the number of drinks per week $\mathrm{x}$ the number of years of drinking (drinks / k x yr) (18). Taking into account the different alcohol concentrations, one drink was considered to correspond to $20 \mathrm{mg}$ of ethanol. Also included were the drinking status, the type of alcoholic beverage, the age at which drinking started, and for former drinkers, the age at which drinking stopped. A characteristic physiological response to drinking alcohol, termed the alcohol flushing response, was also determined. Flushing responses are clinically useful as a simple, cost-effective and non-invasive method for identification of aldehyde dehydrogenase-2 (ALDH2) deficient patients (23). Flushing responses were determined by recording of facial flushing, nausea and tachycardia.

Immunohistochemical staining. The following primary antibodies at the indicated dilutions were used for immunohistochemical staining of $4 \mu \mathrm{m}$ thick paraffin-embedded sections: Rabbit polyclonal anti-Fhit antibody (1:100; clone F130; catalog no. 18163; Immuno-Biological Laboratories, Gunma, Japan), mouse monoclonal anti-p53 (1:50; clone DO-7; catalog no. M7001; Dako; Agilent Technologies, Inc., Santa Clara, CA, USA) or anti-E-cadherin (1:50; clone HECD-1; catalog no. M106; Takara, Bio Inc., Otsu, Japan) antibody and rat monoclonal anti-AID antibody (1:100; clone EK2 5G9; catalog no. 4959; Cell Signaling Technology, Inc., Danvers, MA, USA). Signals were detected using the avidin-biotin-peroxidase complex technique.

The sections were deparaffinized in xylene and rehydrated in graded ethanol series for $5 \mathrm{~min}$ at room temperature. Sections were then immersed in citrate buffer (0.01 M, pH 6.0) and antigens were retrieved by heating in a microwave oven for 20-30 min. Endogenous peroxidase activity was subsequently blocked by incubation with $3 \%$ $\mathrm{H}_{2} \mathrm{O}_{2}$. The sections were then incubated with the primary antibody, or with the same dilution of negative control [normal serum Immunoglobulin $\mathrm{G}(\mathrm{IgG})$ ] overnight at $4^{\circ} \mathrm{C}$. Signals were detected using the Vectastain Elite ABC kit (Vector Laboratories, Burlingame, CA, USA) according to the manufacturer's protocol as follows. The sections were incubated with the appropriate secondary antibody either biotinylated anti-rabbit (catalog no. BA-1000), anti-mouse (catalog no. BA-2000) or anti-rat IgG (catalog no. BA-4000) and with avidin-biotin-peroxidase. The secondary antibodies were purchased from Vector Laboratories (Burlingame, CA, USA), and dilutions of 1:200 were used. Signals were subsequently visualized using diaminobenzidine tetrahydrochloride as a chromogen, and hematoxylin as a counterstain. The expression of proteins was evaluated by two observers, who were blinded to the clinical information with a light microscope.

Assessment of p53 immunostaining. Two criteria were used to define p53 positive staining: A distinct nuclear p53 immunoreaction and p53 staining of $>30 \%$ of tumor cells.

Assessment of Fhit immunostaining. The intensity of Fhit cytoplasmic staining was used to grade Fhit expression as reduced, absent or positive, as previously described (24). The adjacent normal squamous epithelium and lymphocytes of germinal centers were used as internal positive controls. Fhit weak-positive lesions demonstrated positive reactivity at a level that was weak compared with that in the normal epithelia, and these lesions were described as reduced. 
Assessment of E-cadherin immunostaining. Cases were categorized as decreased or negative for E-cadherin expression if they demonstrated definite membrane staining of E-cadherin in $<30 \%$ of the tumor cells, or a complete absence of membrane staining. All other cases were categorized as normal for E-cadherin expression.

Assessment of AID immunostaining. The internal positive controls comprised lymphocytes of germinal centers in lymphoid follicles, since they were mostly activated B cells and all specimens were intensely stained for AID. Cytoplasm was deemed positive when stained at least to the same degree as germinal centers.

Statistical analysis. Data were statistically analyzed using the $\chi^{2}$ test with Yates' correction, the Fisher's test and the Mann-Whitney U-test. Stat Flex version 6.0 (Artech Co., Ltd, Osaka, Japan) was used for all statistical computations. $\mathrm{P}<0.05$ was considered to indicate a statistically significant difference.

\section{Results}

Patient background and clinicopathological features. Backgrounds of the 8 investigated patients with HPSCC are presented in Table I. The patients were all Japanese males, ranging in age from 53 to 77 years (mean, 68.7 years; median, 72.5 years). All patients with HPSCC had a flushing response, along with drinking and smoking habits. The mean DI and BI value of the 8 HPSCC patients were 1,015 and 937.5, respectively. Clinicopathological characteristics and immunostaining results of the investigated 9 HPSCCs and 9 DESCCs from the 8 HPSCC patients are summarized in Table II. Based on a histological examination, the 9 HPSCCs were classified as 6 carcinoma in situ (CIS) and 3 subepithelial invasion. The 9 DESCCs were classified as 6 CIS, 2 carcinoma invading the lamina propria and 1 carcinoma with submucosal invasion. The average maximum diameter of HPSCCs and DESCCs was $14.0 \pm 6.3$ and $22.0 \pm 13.7 \mathrm{~mm}$, with a range of 3 to $25 \mathrm{~mm}$ and 12 to $55 \mathrm{~mm}$, respectively.

Immunohistochemical analysis of p53, Fhit, E-cadherin and AID in HPSCC and DESCC. Table II presents the expression of p53, Fhit, E-cadherin and AID in the endoscopically resected HPSCCs and their DESCCs. Representative immunohistochemical stainings are shown in Fig. 1. Immuno-histochemical staining detected overexpression of p53 and loss of Fhit expression in $8(89 \%)$ and $8(89 \%)$ of the 9 HPSCCs, and in $8(89 \%)$ and $8(89 \%)$ of the 9 DESCCs, respectively, showing a high frequency of such expression. Aberrant E-cadherin and AID expression was identified in $4(44 \%)$ and $6(67 \%)$ of the 9 HPSCCs and in 4 (44\%) and 4 (44\%) of the 9 DESCCs, respectively (Table III). Aberrant expression of p53 and Fhit was identified in 7 tumors out of 9 HPSCCs and in 7 tumors out of their DESCCs (Table IV). In addition, p53 expression was not significantly associated with AID expression $(\mathrm{P}=0.86$; Table V). In univariate analyses, no significant correlation was identified between the expression levels of these proteins and various clinicopathological parameters, including age, sex, size of tumor and location.
Table I. Patient background of superficial hypopharyngeal squamous cell carcinoma cases.

\begin{tabular}{lc}
\hline & $\begin{array}{c}\text { Superficial } \\
\text { hypopharyngeal } \\
\text { squamous cell } \\
\text { carcinoma } \\
\text { cases (n=8) }\end{array}$ \\
Characteristic & $8: 0$ \\
Gender (male:female) & $68.7 \pm 8.8$ \\
Age, years (mean \pm standard deviation) & $8(100 \%)$ \\
Regular alcohol intake & $1015 \pm 531$ \\
Drinking index (mean \pm standard deviation) & $8(100 \%)$ \\
Alcohol flushing & $8(100 \%)$ \\
Current or previous smoker & $937 \pm 849$ \\
Brinkman index (mean \pm standard deviation) & $6(75 \%)$ \\
History of esophageal cancer & \\
\hline
\end{tabular}

\section{Discussion}

It is well established that the multiple primary SCC and the widespread epithelial oncogenic alterations that develop in the head and neck region and the esophagus can be attributed to the field cancerization phenomenon $(1,2)$. NBI combined with magnifying endoscopy has recently been shown to be a useful method for the identification of superficial SCC in the head and neck region as well as in the esophagus (6). In addition, peroral endoscopic resection of superficial HPSCC has also been suggested as a practicable and useful treatment for such tumors $(7,8)$. The present study investigated clinicopathological and biological features of endoscopically resected primary HPSCCs and their DESCCs, which were detected by NBI endoscopy with magnification and/or Lugol chromoendoscopy. To assess biological properties, the expression of p53, Fhit, E-cadherin and AID was immunohistochemically examined. A high frequency of aberrant p53 and Fhit expression was observed in HPSCCs and DESCCs. Furthermore, the status of p53 and Fhit expression in HPSCCs and their DESCCs demonstrated a close similarity, indicating field carcinogenesis had occurred.

Lifestyle factors including heavy smoking and excessive consumption of alcohol have been associated with an increased risk of HNC and esophageal cancer (2). It has been demonstrated for East Asian drinkers that there is a strong association between inactive ALDH2 and the risk of HNC and esophageal cancer $(23,25)$. The presence of inactive ALDH2 can be predicted based on facial flushing in Japanese men aged 40 years or older. In the present study, all patients were males with facial flushing who had drinking and smoking habits.

Alterations in the p53 and FHIT genes and their expression have been reported in a variety of epithelial tumors and premalignant lesions including in HNC and esophageal cancer $(1,2)$. In the present study, a high frequency of aberrant p53 and Fhit expression in HPSCCs and in their DESCCs was observed. Additionally, the pattern of p53 and Fhit expression in the DESCCs closely paralleled that in their HPSCCs. These findings may be compatible with the exposure of the patients to a common carcinogen, such as smoking and drinking habits, 
Table II. Clinicopathological characteristics and immunostaining results of HPSCCs and their DESCCs.

\begin{tabular}{|c|c|c|c|c|c|c|c|c|c|c|}
\hline $\begin{array}{l}\text { Patient } \\
\text { no. }\end{array}$ & $\begin{array}{l}\text { Age, } \\
\text { years }\end{array}$ & Gender & Tumor & $\begin{array}{c}\text { Metachronous/ } \\
\text { synchronous }\end{array}$ & $\begin{array}{l}\text { Diameter, } \\
\mathrm{mm}\end{array}$ & Depth & p53 & Fhit & E-ca & AID \\
\hline \multirow[t]{2}{*}{1} & \multirow[t]{2}{*}{73} & \multirow[t]{2}{*}{ Male } & HPSCC & & 20 & SE & $\mathrm{AE}$ & $\mathrm{AE}$ & $\mathrm{NE}$ & $\mathrm{AE}$ \\
\hline & & & HPSCC & Metachronous & 10 & EP & $\mathrm{AE}$ & $\mathrm{AE}$ & $\mathrm{AE}$ & $\mathrm{AE}$ \\
\hline \multirow[t]{4}{*}{2} & \multirow[t]{4}{*}{75} & \multirow[t]{4}{*}{ Male } & HPSCC & & 15 & EP & $\mathrm{AE}$ & $\mathrm{AE}$ & $\mathrm{AE}$ & $\mathrm{AE}$ \\
\hline & & & DESCC & Metachronous & 14 & EP & $\mathrm{AE}$ & $\mathrm{AE}$ & NE & $\mathrm{NE}$ \\
\hline & & & DESCC & Metachronous & 12 & EP & $\mathrm{AE}$ & $\mathrm{AE}$ & NE & $\mathrm{NE}$ \\
\hline & & & DESCC & Metachronous & 15 & EP & $\mathrm{AE}$ & $\mathrm{AE}$ & $\mathrm{AE}$ & $\mathrm{AE}$ \\
\hline \multirow[t]{2}{*}{3} & \multirow[t]{2}{*}{75} & \multirow[t]{2}{*}{ Male } & HPSCC & & 10 & $\mathrm{EP}$ & $\mathrm{AE}$ & $\mathrm{AE}$ & $\mathrm{AE}$ & $\mathrm{NE}$ \\
\hline & & & DESCC & Synchronous & 55 & SM & $\mathrm{AE}$ & $\mathrm{AE}$ & $\mathrm{AE}$ & $\mathrm{NE}$ \\
\hline 4 & 72 & Male & HPSCC & & 14 & SE & $\mathrm{AE}$ & $\mathrm{NE}$ & $\mathrm{NE}$ & $\mathrm{NE}$ \\
\hline 5 & 60 & Male & HPSCC & & 17 & EP & $\mathrm{AE}$ & $\mathrm{AE}$ & $\mathrm{NE}$ & $\mathrm{AE}$ \\
\hline 6 & 53 & Male & HPSCC & & 25 & EP & $\mathrm{AE}$ & $\mathrm{AE}$ & $\mathrm{NE}$ & $\mathrm{AE}$ \\
\hline \multirow[t]{4}{*}{7} & \multirow[t]{4}{*}{77} & \multirow[t]{4}{*}{ Male } & HPSCC & & 3 & SE & $\mathrm{AE}$ & $\mathrm{AE}$ & NE & $\mathrm{NE}$ \\
\hline & & & DESCC & Synchronous & 30 & LPM & $\mathrm{AE}$ & $\mathrm{AE}$ & $\mathrm{AE}$ & $\mathrm{AE}$ \\
\hline & & & DESCC & Synchronous & 15 & EP & $\mathrm{AE}$ & $\mathrm{NE}$ & $\mathrm{NE}$ & $\mathrm{AE}$ \\
\hline & & & DESCC & Synchronous & 20 & EP & $\mathrm{AE}$ & $\mathrm{AE}$ & $\mathrm{NE}$ & $\mathrm{AE}$ \\
\hline \multirow[t]{3}{*}{8} & \multirow[t]{3}{*}{62} & \multirow[t]{3}{*}{ Male } & HPSCC & & 12 & EP & $\mathrm{NE}$ & $\mathrm{AE}$ & $\mathrm{AE}$ & $\mathrm{AE}$ \\
\hline & & & DESCC & Synchronous & 13 & $\mathrm{EP}$ & $\mathrm{NE}$ & $\mathrm{AE}$ & $\mathrm{AE}$ & $\mathrm{NE}$ \\
\hline & & & DESCC & Metachronous & 24 & LPM & $\mathrm{AE}$ & $\mathrm{AE}$ & $\mathrm{NE}$ & $\mathrm{NE}$ \\
\hline
\end{tabular}

HPSCC, hypopharyngeal squamous cell carcinoma; DESCC, double esophageal squamous cell carcinoma; Fhit, Fragile Histidine Triad; E-ca, E-cadherin; AID, activation-induced cytidine deaminase; SE, carcinoma with subepithelial invasion; AE, aberrant expression; NE, normal expression; EP, carcinoma in situ; SM, carcinoma with submucosal invasion; LPM, carcinoma invading the lamina propria.

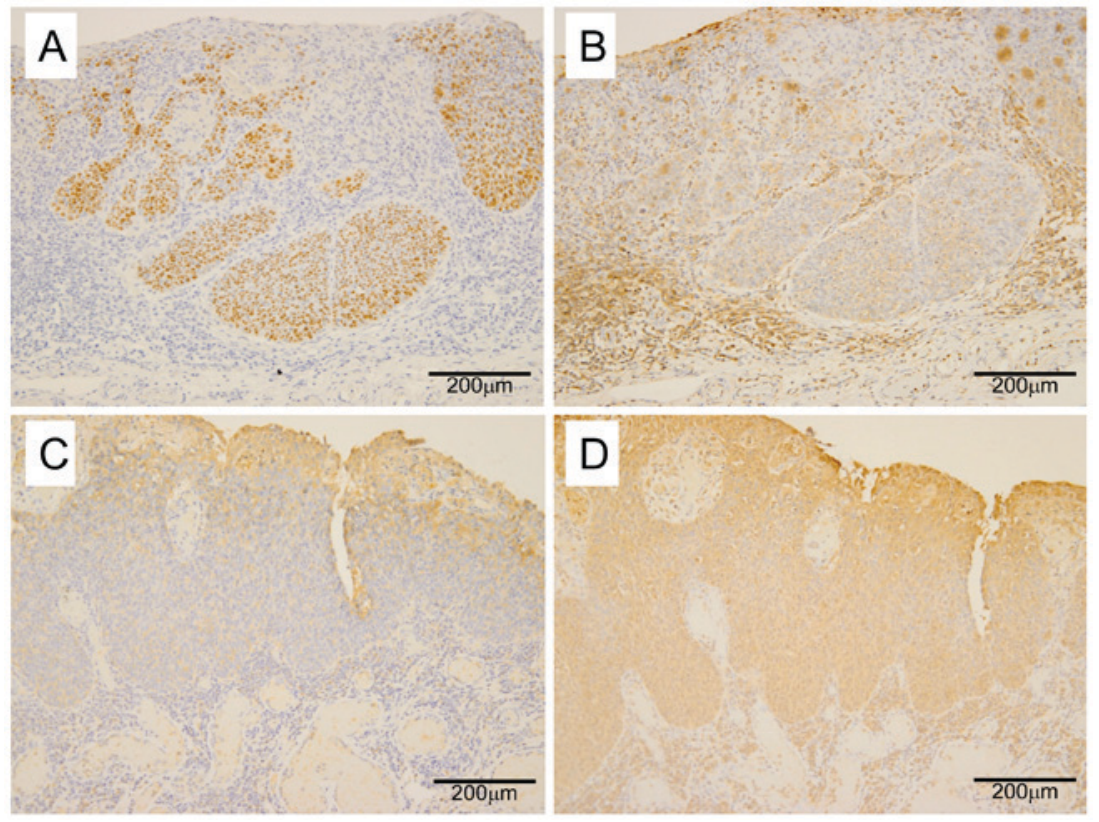

Figure 1. Representative results of p53, Fhit, E-cadherin and AID immunostaining in HPSCC tissue. (A) Positive p53 immunostaining of CIS in hypopharyngeal cancer. (B) Reduced Fhit immunostaining of CIS and positive staining of the germinal center and infiltrating lymphocytes. (C) Reduced E-cadherin immunostaining of CIS and decreased membrane staining of E-cadherin in <30\% of the tumor cells. (D) Positive AID immunostaining of CIS in hypopharyngeal cancer. Scale bar, $200 \mu \mathrm{m}$. Magnification, x200. Fhit, Fragile Histidine Triad; AID, activation-induced cytidine deaminase; HPSCC, hypopharyngeal squamous cell carcinoma; CIS, carcinoma in situ.

from the viewpoint of field cancerization. The frequency of aberrant p53 and Fhit expression was increased compared with what has been observed in previous reports $(1,2,26,27)$. The present data were obtained from endoscopically resected, 
Table III. Summary of analysis of p53, Fhit, E-cadherin and AID protein expression in superficial HPSCCs and their DESCCs.

\begin{tabular}{lcccc}
\hline Type & $\begin{array}{c}\mathrm{p} 53 \\
(\%)\end{array}$ & $\begin{array}{c}\text { Fhit } \\
(\%)\end{array}$ & $\begin{array}{c}\text { E-cadherin } \\
(\%)\end{array}$ & $\begin{array}{c}\text { AID } \\
(\%)\end{array}$ \\
\hline HPSCC $(\mathrm{n}=9)$ & $8(89)$ & $8(89)$ & $4(44)$ & $6(67)$ \\
$\operatorname{DESCC}(\mathrm{n}=9)$ & $8(89)$ & $8(89)$ & $4(44)$ & $4(44)$ \\
\hline
\end{tabular}

Fhit, Fragile Histidine Triad; AID, activation-induced cytidine deaminase; HPSCC, hypopharyngeal squamous cell carcinoma; DESCC, double esophageal squamous cell carcinoma.

Table IV. Association between p53 and Fhit expression in superficial HPSCCs and DESCCs.

\begin{tabular}{lcc}
\hline Protein expression & HPSCC $\mathrm{n}=9$ & DESCC $\mathrm{n}=9$ \\
\hline p53(-)/Fhit(+) & 0 & 0 \\
p53(-)/Fhit(-) & 1 & 1 \\
p53(+)/Fhit(+) & 1 & 1 \\
p53(+)/Fhit(-) & 7 & 7 \\
\hline
\end{tabular}

Fhit, Fragile Histidine Triad; HPSCC, hypopharyngeal squamous cell carcinoma; DESCC, double esophageal squamous cell carcinoma; -, loss of expression; + , positive for expression.

Table V. Association between p53 and AID expression in superficial HPSCCs and DESCCs.

$$
\text { p53 }
$$

\begin{tabular}{lccc}
\cline { 2 - 4 } AID & Positive & Negative & Total \\
\hline Positive & 9 & 1 & $10^{\mathrm{a}}$ \\
Negative & 7 & 1 & $8^{\mathrm{a}}$ \\
Total & 16 & 2 & 18
\end{tabular}

${ }^{\text {a }} \mathrm{P}=0.86$ by the $\chi^{2}$ test with Yates' correction. AID, activation-induced cytidine deaminase; HPSCC, hypopharyngeal squamous cell carcinoma; DESCC, double esophageal squamous cell carcinoma.

early carcinoma. Therefore, the staging of the present cases differed from those of previous reports. However, the present data are consistent with previous reports that p53 protein overexpression is closely linked with the multiplicity of esophageal cancer and with the development of multiple primary cancers $(28,29)$. Therefore, the p53 and Fhit alterations observed in patients clearly associated with field cancerization may frequently occur in the early stages of head, neck and esophageal carcinogenesis. The biological nature of HPSCC based on the p53 and Fhit status may share strong similarities with that of their double ESC. Based on the above data the present study considered that patients at high risk of having
DESCCs may by usefully screened by p53 and Fhit expression and that the results of such screening may have value for directing the clinical management of patients with HNCs.

Complete loss or reduced expression of E-cadherin was previously detected in patients with invasive HNC $(60 \%$ of patients) and invasive ESCC (42.3\% of patients) $(9,30)$. In addition, loss of E-cadherin expression in HNC and ESCC is associated with tumor invasiveness, metastasis and prognosis $(9,20)$. Although the present study analyzed early stage tumors, it demonstrated a similar frequency of E-cadherin loss/reduced expression compared with several published studies (9,30). Aberrant AID expression induces p53 gene mutation in Helicobacter pylori infected gastric epithelial cells and is associated with the development of precancerous lesions and the progression of malignant tumors (12). We previously detected aberrant AID expression in $33-45 \%$ of early esophageal squamous neoplasia (27). This aberrant AID expression rate was similar to that detected in the present study. In our previous study, we proposed that $\mathrm{p} 53$ expression was independent of aberrant AID expression in the early stage of esophageal carcinogenesis (27). Similarly, the present study identified no association between aberrant AID expression and p53 overexpression in the superficial HPSCCs and their DESCC samples. The molecular mechanism underlying AID upregulation in hypopharyngeal and esophageal tissues is unclear. Previous studies have indicated that AID expression does perform an important role in the early stages of oral carcinogenesis and that AID expression in oral squamous cells is induced in response to inflammatory cytokine stimulation $(31,32)$. Additionally, the frequencies of aberrant E-cadherin and AID expression were similar in HPSCCs and DESCCs, although their expression did not correlate with each other. These results may also be due to the field effect of tobacco and alcohol.

In conclusion, the frequency and pattern of aberrant p53, Fhit, E-cadherin, and AID expression in the examined superficial HPSCCs and their DESCCs was consistent with the field theory of cancerization. Furthermore, it is proposed that p53 and Fhit expression may be candidate biomarkers for predicting the development of DESCC in patients with HPSCC. A knowledge of the aberrant expression of these genes could also provide a basis for improving the strategies used for the early endoscopic detection and treatment of early stage HPSCC and ESCC.

\section{References}

1. Perez-Ordoñez B, Beauchemin M and Jordan RC: Molecular biology of squamous cell carcinoma of the head and neck. J Clin Pathol 59: 445-453, 2006.

2. Toh Y, Oki E, Ohgaki K, Sakamoto Y, Ito S, Egashira A, Saeki H, Kakeji Y, Morita M, Sakaguchi Y, et al: Alcohol drinking, cigarette smoking, and development of squamous cell carcinoma of the esophagus: Molecular mechanisms of carcinogenesis. Inc J Clin Oncol 15: 135-144, 2010.

3. Slaughter DP, Southwick HW and Smejkal W: Field cancerization in oral stratified squamous epithelium; clinical implications of multicentric origin. Cancer 6: 963-968, 1953.

4. Ina H, Shibuya H, Ohashi I and Kitagawa M: The frequency of a concomitant early esophageal cancer in male patients with oral and oropharyngeal cancer. Screening results using Lugol dye endoscopy. Cancer 73: 2038-2041, 1994.

5. Dubuc J, Legoux J-, Winnock M, Seyrig J-, Barbier J-, Barrioz T, Laugier R, Boulay G, Grasset D, Sautereau D, et al: Endoscopic screening for esophageal squamous cell carcinoma in high-risk patients: A prospective study conducted in 62 French endoscopy centers. Endoscopy 57: 690-695, 2006. 
6. Goda K, Dobashi A and Tajiri H: Perspectives on narrow-band imaging endoscopy for superficial squamous neoplasms of the orohypopharynx and esophagus. Dig Endosc 26 (Suppl 1): S1-S11, 2014

7. Shimizu Y, Yamamoto J, Kato M, Yoshida T, Hirota J Ono Y, Nakagawa M, Nakagawa S, Oridate $\mathrm{N}$ and Asaka M: Endoscopic submucosal dissection for treatment of early stage hypopharyngeal carcinoma. Gastrointest Endosc 64: 255-262, 2006.

8. Hanaoka N, Ishihara R, Takeuchi Y, Suzuki M, Otozai S, Kida K, Yoshii T, Fujii T, Yoshino K, Sugawa T, et al: Endoscopic submucosal dissection as minimally invasive treatment for superficial pharyngeal cancer: A phase II study (with video). Gastrointest Endosc 82: 1002-1008, 2015.

9. Mattijssen V, Peters HM, Schalkwijk L, Manni JJ, van't Hof-Grootenboer B, de Mulder PH and Ruiter DJ: E-cadherin expression in head and neck squamous-cell carcinoma is associated with clinical outcome. Int J Cancer 55: 580-585, 1993.

10. Xu XC: Risk factors and gene expression in esophageal cancer. Methods Mol Biol 471: 335-360, 2009.

11. Muramatsu M, Kinoshita K, Fagarasan S, Yamada S, Shinkai Y and Honjo T: Class switch recombination and hypermutation require activation-induced cytidine deaminase (AID), a potential RNA editing enzyme. Cell 102: 553-563, 2000.

12. Matsumoto Y, Marusawa H, Kinoshita K, Endo Y, Kou T, Morisawa T, Azuma T, Okazaki IM, Honjo T and Chiba T: Helicobacter pylori infection triggers aberrant expression of activation-induced cytidine deaminase in gastric epithelium. Nat Med 13: 470-476, 2007.

13. Hollestein M, Sidransky D, Vogelstein B and Harris CC: p53 mutations in human cancers. Science 253: 49-53, 1991.

14. Ohta M, Inoue H, Cotticelli MG, Kastury K, Baffa R, Palazzo J, Siprashvili Z, Mori M, McCue P, Druck T, et al: The FHIT gene, spanning the chromosome 3 p14.2 fragile site and renal carcinoma-associated $\mathrm{t}(3 ; 8)$ breakpoint, is abnormal in digestive tract cancer. Cell 84: 587-597, 1996.

15. Mori M, Mimori K, Shiraishi T, Alder H, Inoue H, Tanaka Y, Sugimachi K, Huebner K and Croce CM: Altered expression of Fhit in carcinoma precarcinomatous lesions of the esophagus. Cancer Res 60: 1177-1182, 2000.

16. Lee EJ, Lee BB, Kim JW, Shim YM, Hoseok I, Han J, Cho EY, Park J and Kim DH: Aberrant methylation of Fragile Histidine Triad gene is associated with poor prognosis in early stage esophageal squamous cell carcinoma. Eur J Cancer 42: 972-980, 2006.

17. Soma T, Kaganoi J, Kawabe A, Kondo K, Imamura $\mathrm{M}$ and Shimada Y: Nicotine induces the fragile histidine triad methylation in human esophageal squamous epithelial cells. Int J Cancer 119: 1023-1027, 2006.

18. Morita M, Oyama T, Nakata S, Ono K, Sugaya M, Uramoto H, Yoshimatsu T, Hanagiri T, Sugio K and Yasumoto K: Expression of FHIT in esophageal epithelium and carcinoma: Reference to drinking, smoking and multicentric carcinogenesis. Anticancer Res 26: 2243-2248, 2006.

19. Ishii H, Dumon KR, Vecchione A, Trapasso F, Mimori K, Alder H, Mori M, Sozzi G, Baffa R, Huebner K and Croce CM: Effect of adenoviral transduction of the fragile histidine triad gene into esophageal cancer cells. Cancer Res 61: 1578-1584, 2001.
20. Tamura S, Shiozaki H, Miyata M, Kadowaki T, Inoue M, Matsui S, Iwazawa T, Takayama T, Takeichi M and Monden M: Decreased E-cadherin expression is associated with haematogenous recurrence and poor prognosis in patients with squamous cell carcinoma of the oesophagus. Br J Surg 83: 1608-1614, 1996.

21. Hirohashi S: Inactivation of the E-cadherin-mediated cell adhesion system in human cancers. Am J Pathol 153: 333-339, 1998.

22. Warren S and Gates O: Multiple primary, malignant tumors: A survey of the literature and statistical study. Am J Cancer 16: 1358-1414, 1932

23. Brooks PJ, Enoch MA, Goldman D, Li TK and Yokoyama A: The alcohol flushing response: An unrecognized risk factor for esophageal cancer from alcohol consumption. PLoS Med 6: e50, 2009.

24. Saldivar JC, Shibata H and Huebner K: Pathology and biology associated with the fragile FHIT gene and gene product. J Cell Biochem 109: 858-865, 2010.

25. Yokoyama A, Mizukami T and Yokoyama T: Genetic polymorphisms of alcohol dehydrogense-1B and aldehyde dehydrogenase-2, alcohol flushing, mean corpuscular volume, and aerodigestive tract neoplasia in Japanese drinkers. Adv Exp Med Biol 815: 265-279, 2015.

26. Sauter ER, Cleveland D, Trock B, Ridge JA and Klein-Szanto AJ: p53 is overexpressed in fifty percent of pre-invasive lesions of head and neck epithelium. Carcinogenesis 15: 2269-2274, 1994.

27. Hayashi A, Yashima K, Takeda Y, Sasaki S, Kawaguchi K, Harada K, Murawaki Y and Ito H: Fhit, E-cadherin, p53, and activation-induced cytidine deaminase expression in endoscopically resected early stage esophageal squamous neoplasia. J Gastroenterol Hepatol 27: 1752-1758, 2012.

28. Kohmura T, Hasegawa Y, Ogawa T, Matsuura H, Takahashi M, Yanagita N and Nakashima T: Cyclin D1 and p53 overexpression predicts multiple primary malignant neoplasms of the hypopharynx and esophagus. Arch Otolaryngol Head Neck Surg 125: 1351-1354, 1999.

29. Kato H, Yoshikawa M, Miyazaki T, Nakajima M, Fukai Y, Tajima K, Masuda N, Tsukada K, Fukuda T, Nakajima T and Kuwano H: Expression of p53 protein related to smoking and alcoholic beverage drinking habits in patients with esophageal cancers. Cancer Lett 167: 65-72, 2001.

30. Chung Y, Lam AK, Luk JM, Law S, Chan KW, Lee PY and Wong J: Altered E-cadherin expression and p120 catenin localization in esophageal squamous cell carcinoma. Ann Surg Oncol 14: 3260-3267, 2007.

31. Miyazaki Y, Fujinami M, Inoue H, Kikuchi K, Ide F and Kusama K: Expression of activation-induced cytidine deaminase in oral epithelial dysplasia and oral squamous cell carcinoma. J Oral Sci 55: 293-299, 2013.

32. Nakanishi Y, Kondo S, Wakisaka N, Tsuji A, Endo K, Murono S, Ito M, Kitamura K, Muramatsu M and Yoshizaki T: Role of activation-induced cytidine deaminase in the development of oral squamous cell carcinoma. PLoS One 8: e62066, 2013. 